\title{
Self-propelling micro-nanorobots: challenges and future perspectives in nanomedicine
}

Nanorobotics: future nanovehicles in drug delivery

Nanorobots are envisioned since decades to be the future of nanomedicine in the active transport of therapeutics to specific locations. Here we comment on the progress that has emerged over the last few years towards that dream, and the challenges that need to be overcome to make it real.

\section{Xing Ma}

Sate Key Laboratory of Advanced Welding and Joinng, Harbin Institute of Technology (Shenzhen), Shenzhen 518055, China

and

Key Laboratory of Micro-systems and Micro-structures Manufacturing of Ministry of Education, Harbin Institute of Technology, Harbin 150001, China

Samuel Sánchez

Institució Catalana de Recerca i Estudis Avancats (ICREA), Pg. Lluís Companys 23, 08010 Barcelona, Spain

and

Institut de Bioenginyeria de Catalunya (IBEC), Baldiri i Reixac 10-12, 08028 Barcelona, Spain ssanchez@ibecbarcelona.eu

Keywords: nanorobots, nanomotors, drug delivery, nanomachines, target delivery 
Micro/nano-motors (MNM) refer to small objects capable of generating autonomous motion in fluids at small scale, typically within micro/nano regime. This growing field is continuously attracting new groups from different disciplines, and moving forwards with fast pace. Up to now, researchers have designed and fabricated new types of micro/nano-motors, more bio-friendly, powerful, and versatile in shapes and materials capable of moving in fluidic environment. Meanwhile, various proofof-concept demonstrations on possible applications of these MNM have been reported, including cargo transportation, chemical/biological sensing, cell and tissue penetration, cell transport, wound healing, environmental remediation, among others. [1,2] Altogether scientists have proposed and made the first attempts to use these active carriers in the field of nanomedicine. The rapid development of active, smart vehicles such as MNM towards biomedical applications has been a research topic of high interest in basic science, technology, medicine and even in the public opinion which we believetheir actual use is not too far in future.[3]

At present, nanoparticles based drug delivery relies on the passive transport of the drug by the different flows encountered when nanoparticles are introduced into living systems. Active transport by using self-propelled nanoparticles (named nanomotors or nanorobots) aims at enhancing the diffusion of the drug to specific locations on demand. Ideally, they will overcome the flows or reach locations where the diffusion is very limited or the drugs cannot even penetrate, for instance where viscosity is very high. Taking advantage of the controlled locomotion of MNM capable of drug loading and releasing, ideally these "intelligent" MNM are expected to realize direct targeted drug delivery in an active manner. Thus, the field of biomedicine is apparently an ideal scenario where self-propelled MNM could develop a large number of applications in the near future. Some proof-of concept reports demonstrated the delivery of anti-cancer drugs by using self-propelled MNM,[4] and even further drug releasing after reaching the target cells in vitro. [5, 6] Although several publications on this topic have been reported, foreseeable gaps between these demonstrations and realistic biomedical use are rather obvious. In order to achieve such advanced active drug delivery vehicle, there are several prerequisites have to be satisfied. The materials of which the MNM is made of must be biocompatible. The fuel providing driving force for the propulsion should be available in the fluid itself, and ideally not added externally (at least not toxic to the organism). Then, the carriers require precise control on the directionality of their movement to reach the desired target, which could be given by external stimuli (magnetic, electric, ultrasound fields) or sensing in-situ gradients $(\mathrm{pH}$, temperature, chemical composition). MNM should have great cargo loading capabilities in terms of weight transported and also on the type of drugs loaded. Furthermore, versatile methods of drug delivery should be considered on demand. In this commentary, we will mainly discuss the biocompatibility issue when using MNM as biomedical carriers.

\section{Fabrication of MNM with biocompatible or biodegradable materials}

When applying any external objects into biological applications, safety issues are the first consideration. In fact, up to now, many kinds of materials have been utilized to fabricate motors. For instance, self-assembly of organic component such as proteins or polymers has been utilized to fabricate bubble propelled tubular and spherical motors which can be biocompatible or biodegradable regarding their motors' body.[7] Another method was the use of materials that can consume the motors' body by chemical reactions such as those made of water or acid-sensitive $\mathrm{Al}$ and $\mathrm{Mg}$, producing bubbles and self-propulsion.[8, 9] These motors' body would be consumed and disappear after finishing their delivery task. Furthermore, our group has developed a series of micro/nano-motors with biocompatible material of mesoporous silica which is a material that has been widely utilized in biomedical field- and proved to be biodegradable under certain conditions. $[10,11]$ We evaluated the MTT cytotoxicity of our MNM in human cell lines (HeLa cells), which showed negligible toxic effect. In spite of initial studies like MTT assay for the toxicity evaluation of the motors' body, due to the early development stage of this field systematic evaluation of the biocompatibility of all these motors' body is still necessary to guarantee the safety of using these motors into real biological use, like in vivo study or clinic test.

Although there are several materials utilized to form the MNM and proven to not affect cell integrity and viability in vitro, the step towards in vivo explorations will definitely require the use of non-toxic fuel to power the self-propulsion of the motors. However, the biocompatibility and in vivo biologically availability of the fuel brings great challenges for the biomedical use of these active 
motors, which will be the major discussion of this article and addressed in detail in the following content.

\section{Self-propulsion of MNM by biocompatible fuels}

Chemically Powered Motors: Moving at micro/nano dimensions is suffering from negligible inertia and high viscosity of fluid when compared to macro-scale machine. Therefore, chemically powered MNM needs to convert chemical energy into kinetic energy by continuously obtaining fuel from the environment. The high power output developed by some MNM, but the toxicity of most frequently used fuel hydrogen peroxide $\left(\mathrm{H}_{2} \mathrm{O}_{2}\right)$ has been a long-standing obstacle hindering any biomedical exploration of MNM. In order to overcome this challenge, researchers explored alternative chemical reactions to replace $\mathrm{Pt} / \mathrm{H}_{2} \mathrm{O}_{2}$ combination, such as $\mathrm{H}_{2}$ bubble generation by water-metal (e.g. All or $\mathrm{Mg}$ ) reactions. [8, 9] Harsh reaction conditions and short life time still limit their extension into biomedical field. Very recently several research groups independently developed self-propelled MNM driven by non-toxic fuel via utilizing enzyme triggered bio-catalytic reactions to power the motors, consuming physiologically available fuels, such as glucose and urea.[10-14] At the nanoscale, most of the enzyme-based nanomotors present enhanced Brownian motion with limited directionality.[1114]Yet, our group achieved urea powered long-range directional phoretic propulsion of biocompatible microcapsule motors which were capable of multiple cargo loading. In addition to biocompatibility of the fuel and motors' body, we succeeded to control the velocity of the motors by chemically manipulating the enzymatic activity based on the reversible enzyme inhibition. Magnetic guidance on the direction of motion was integrated into our system as well.[10] These achievements have provided exciting expectations on the further explorations about the in vivo biomedical use of these enzyme powered MNM.

External field driven $M N M$ can avoid the requirement of availability of fuel from surrounding environment compared to chemically powered MNM. Instead, they acquire their kinetic driving force by reacting to externally applied fields, including magnetic, electric, light, ultrasound, etc. Furthermore, one advantage of external field driven MNM is that their motion is not affected by the ionic media. For instance, researchers developed micro/nano-propeller featured with helix structures by systematic fabrication methods, which were driven by an external magnetic field that induced their rotation, achieving "drilling and advancing" in aqueous solution.[15, 16] Aiming at more realistic situation for biomedical use, the motion behavior of these helix propellers in complex viscoelastic media was investigated.[17] However, further demonstrations are needed to show the advantages of self-propelled carriers when compared to simple magnetic attraction.

Another frequently used external field is ultrasound, which is well known and widely utilized in medical field. The transmission of acoustic waves in human body is generally safe with proper wavelength and power input. Researchers used ultrasound to propel MNM, such as metallic nanorods and nanowires by fuel-free mechanism. Due to biocompatibility of ultrasound, researchers forwarded the ultrasound propelled nano-rods into in vitro test[18] and even the siRNA delivery for green florescence protein silence was reported.[19] The stochastic self-propelled motion makes it less usable for other biomedical applications as an active carrier for targeted delivery. However, the integration with magnetic nanoparticles into ultrasound powered motors (e.g. red blood cell motors) could provide directionality control by external magnetic field, and enable their potentials for targeted drug delivery.[20]

Light powered MNM are advantageous when considering the easy control on the energy input by tuning the light power. For the photo-catalytic powered MNM based on semiconductors such as $\mathrm{TiO}_{2}$,[21, 22] the use of biologically harmful ultraviolet (UV) light or addition of $\mathrm{H}_{2} \mathrm{O}_{2}$ fuel still hampers its advancement towards biomedical field. Another mechanism is based on photo-thermal effect by using near infrared (NIR) light to produce heat at one side of Janus motors coated with gold and induce self-thermophoresis.[23] However, such system requires relatively high light power, bringing new challenges for further exploration on in vivo use.

\section{Challenges and Outlook}

Although there have been considerable efforts on this research topic with significant advancement in recent years, it is still a great challenge to fabricate a self-propelled system that can fulfill all the requirements of realistic biomedical task in vitro or in vivo. Based on the current progress 
of biomedical applications of MNM, most of reports were mainly proof-of-concept demonstrations, such as aiming at active targeted drug delivery and transient or uncontrolled active motion in biological environment.

Regarding chemically powered MNM, the most promising system is enzyme powered MNM, since we can fabricate biocompatible motors' body and using physiologically available fuel to power the motors. In addition to typical spherical Janus motors in which case enzymes were asymmetrically immobilized on one side of the external surface of a particle or nanorods to provide the driving force, $[11,12,14]$ researchers also confined enzymes in supramolecular stomatocyte and achieved selfpropulsion.[13] The motion mechanism of these enzyme powered motors is still intrigue, and will require further understanding for its advancement towards biomedical use. In particular, the most promising system capable of long-range self-propulsion is generally accepted to be powered by phoretic mechanism which showed sensitivity towards ionic strength. The cease of motion with electrolytes at millimolar range makes these enzyme motors useless when considering hundreds of millimolar electrolytes concentration in biological fluid. In combination with noble metal, e.g. Pt and $\mathrm{Au}$, the photo-catalytic reactions to powered motors could move in pure water under light exposure, but its active motion also suffers from ionic effect. Although we achieved the development of motors that can be powered by non-toxic fuel, for reasons still not fully understood, their motion mechanism is not feasible within the biological environment. Therefore, future research should carry out in-depth investigations on the fundamental understanding on the phoretic motion of these bio-friendly fuel driven motors, in the hope of developing chemically powered motors capable of overcoming ionic effect. Then, that will be a stepping stone towards any biomedical research, such as targeted transportation of anti-cancer drugs towards tumor site in vivo.

For external field powered MNM based on fuel free propulsion mechanism, their motion does not halt with the ionic effect. Besides similar challenges given by viscosity and complexity of the real biological fluid that hinders chemically powered MNM, another easily foreseeable difficulty for external field powered MNM is the challenge of precisely applying external field to provide continuous propulsion force in vivo. For instance, tissue penetration and light attenuation might affect the performance of light powered motors for in vivo applications. And in vivo light introduction and continuous light tracking on the moving motors will bring further challenges as well. The external field powered MNM usually requires a complicated and accurate location of the field applied on the position of the motors, which will surely bring more obstacles towards realistic use of these motors in human body.

Besides these challenges with the motors' motion capability in vivo, the other side of the coin is the influencing factors from the realistic physiological environment. First of all, for active carriers, these MNM are supposed to shuttle within blood vessels, where strong hydrodynamic flow stream of the blood will strongly affect the localized flow field around the MNM, which is crucial for the selfpropulsion of the MNM. For instance, the phoretic motion mechanism of motors propelled by asymmetric reaction generated chemical species (neutral or ionic) concentration gradient will be significantly altered. Furthermore, there will be an extremely high possibility of interactions between the MNM and micro/nano-size objects in the blood, such as red blood cells, proteins, etc., including non-specific binding or collisions, which might affect the catalytic/bio-catalytic activities of the selfpropulsion systems in chemically powered MNM. So, it will introduce more difficulties for the selfpropulsion capability, as well as motion control in real bio-environment.

Therefore, instead of directly heading for proof-of-concept demonstrations of potential biomedical applications of a newly developed MNM, more fundamental studies are highly demanded to explore the biomedical potential of these MNM from the root. Regarding this aspect, it is necessary to carry out investigations on the static interaction between real bio-fluid with potential biomedical MNM. It is also recommended to explore the motion behavior of MNM in highly viscous media, simulating specific bio-environment like the joints, where viscosity is high and diffusion of medicine is a challenge. The active motion of MNM might help to deliver drugs into these areas where the diffusion of drugs is limited. Another possible direction might be a different view from outside of current goals for biomedical use of MNM, in addition to the most commonly explored purpose of active targeted drug delivery. And interdisciplinary interactions with other fields are highly recommended, especially with researchers or clinicians, which might be able to inspire new biomedical applications of MNM, by making good use of its small dimensions and active motion capability. 


\section{Conclusion}

In summary, the development of MNM has experienced a fast growth within the past decade and quickly expanded into a variety of different applications. In particular, biomedical use of MNM has been a long-chased scientific dream ever since the first invention. Up to now, considerable efforts have been put into this research topic and we have achieved significant progress regarding to the biocompatibility of the motors' body, as well as substituting the toxic fuel with non-toxic and physiologically available fuel. External field powered propulsion systems are fuel-free and possess advantages of remote control capability while powering them. The complexity of their propulsion behaviour and future motion control will be challenges to be tackled to the biomedical use of MNM. Yet, for all of them, the study of collective motion will be of high importance as MNM will need to act collectively for useful applications in medicine. Although a large number of proof-of-concept demonstrations of biomedical applications of MNM have been reported, there are only a few realistic micro/nano-propulsion systems that can be utilized in vitro or in vivo. Therefore, the fundamental propulsion mechanism of chemically powered MNM should be revealed and understood, and further development of new propulsion systems in real bio-fluid is required. Comparative investigations of the passive versus active particles to demonstrate the drug delivery efficiency of the MNM should be carried out as well. On the other hand, synergy from multiple research fields are necessary to find out some crucial biomedical application of the self-propelled MNM, which will make these intelligent micro/nano devices powerful tools in future nanomedicine field.

\section{Reference}

1. Sánchez S, Soler L, Katuri J. Chemically powered micro- and nanomotors. Angew. Chemie Int. Ed. 54(5), 1414-1444 (2015).

2. Wang H, Pumera M. Fabrication of micro/nanoscale motors. Chem. Rev. 115(16), 8704-8735 (2015).

3. Abdelmohsen L, Peng F, Tu YF, Wilson DA. Micro- and nano-motors for biomedical applications. J. Mater. Chem. B 2(17), 2395-2408 (2014).

4. Ma X, Hahn K, Sanchez S. Catalytic mesoporous Janus nanomotors for active cargo delivery. J. Am. Chem. Soc. 137(15), 4976-4979 (2015).

5. Tu Y, Peng F, André AaM, Men Y, Srinivas M, Wilson DA. Biodegradable hybrid stomatocyte nanomotors for drug Delivery. ACS Nano 11(2), 1957-1963 (2017).

6. Wu Y, Lin X, Wu Z, Möhwald H, He Q. Self-propelled polymer multilayer Janus capsules for effective drug delivery and light-triggered release. ACS Appl. Mater. Interfaces 6(13), 1047610481 (2014).

7. Lin X, Wu Z, Wu Y, Xuan M, He Q. Self-propelled micro-/nanomotors based on controlled assembled architectures. Adv. Mater. 28(6), 1060-1072 (2016).

8. Gao W, Pei A, Wang J. Water-driven micromotors. ACS Nano 6(9), 8432-8438 (2012).

9. Mou FZ, Chen CR, Ma HR, Yin YX, Wu QZ, Guan JG. Self-propelled micromotors driven by the magnesium-water reaction and their hemolytic properties. Angew. Chemie Int. Ed. 52(28), 7208-7212 (2013).

10. Ma X, Wang X, Hahn K, Sánchez S. Motion control of urea-powered bBiocompatible hollow microcapsules. ACS Nano 10(3), 3597-3605 (2016).

11. Ma X, Jannasch A, Albrecht U-R et al. Enzyme-powered hollow mesoporous Janus nanomotors. Nano Lett. 15(10), 7043-7050 (2015).

12. Dey KK, Zhao X, Tansi BM et al. Micromotors powered by enzyme catalysis. Nano Lett. 15(12), 8311-8315 (2015).

13. Abdelmohsen LKEA, Nijemeisland M, Pawar GM et al. Dynamic loading and unloading of proteins in polymeric stomatocytes: fFormation of an enzyme-loaded supramolecular nanomotor. ACS Nano 10(2), 2652-2660 (2016).

14. Bunea A-I, Pavel I-A, David S, Gáspár S. Sensing based on the motion of enzyme-modified nanorods. Biosens. Bioelectron. 67, 42-48 (2015).

15. Ghosh A, Fischer P. Controlled propulsion of artificial magnetic nanostructured propellers. Nano Lett. 9(6), 2243-2245 (2009).

16. Tottori S, Zhang L, Qiu F, Krawczyk KK, Franco-Obregón A, Nelson BJ. Magnetic helical micromachines: fabrication, controlled wwimming, and cargo transport. Adv. Mater. 24(6), 811-816 (2012).

17. Schamel D, Mark AG, Gibbs JG et al. Nanopropellers and their actuation in complex 
viscoelastic media. ACS Nano 8(9), 8794-8801 (2014).

18. Wang W, Li S, Mair L, Ahmed S, Huang TJ, Mallouk TE. Acoustic propulsion of nanorod motors inside living cells. ngew. Chemie Int. Ed. 126(12), 3265-3268 (2014).

19. Esteban-Fernández De vila B, Angell C, Soto F et al. Acoustically propelled nanomotors for intracellular siRNA delivery. ACS Nano 10(5), 4997-5005 (2016).

20. Wu Z, Li T, Li J et al. Turning erythrocytes into functional micromotors. ACS Nano 8(12), 12041-12048 (2014).

21. Chen C, Mou F, Xu L et al. Light-steered isotropic semiconductor micromotors. Advanced Materials 29(3), (2017). DOI: 10.1002/adma.201603374

22. Dai B, Wang J, Xiong $\mathrm{Z}$ et al. Programmable artificial phototactic microswimmer. Nat. Nanotechnol. 11(12), 1087-1092 (2016).

23. Xuan M, Wu Z, Shao J, Dai L, Si T, He Q. Near infrared light-powered Janus mesoporous silica nanoparticle motors. J. Am. Chem. Soc. 138(20), 6492-6497 (2016). 